\title{
A New Technique For Visualizing Smart Substation IED Interactive Processes
}

\author{
Tangzhijun $^{1}$, Zhaibolong $^{1}$ Linguodong $^{1}$, Chaowujie $^{1}$ \\ 1.State Grid Fujian Electric Power Company Research Institute, Fuzhou, 350007, China \\ 13705004007@139.com,zhaibolong@163.com,chaowujie1013@163.com
}

Keywords : IEC61850; Smart Substation; IED; Data

Interaction; Visualization

\begin{abstract}
This paper presents an innovative technique of the IED interactive process visual analysis in the IEC61850 based smart substation. By parsing the data from the substation SCD configuration file to extract the data model needed for graphical dynamic analysis, the solution acquires the real-time communication data of substation according to the SMV, GOOSE and MMS messages. Using Java2D technology, the solution interprets the information in a visual way of expression to generate time-sequentially progressive display automatically. Such graphical representation forms a complete record of the actions, and provides a simple and clear analyzing view of a complex and difficult to understand process.
\end{abstract}

\section{Introduction}

\subsection{Industrial Background}

The introduction of IEC61850 based smart substation brings many technological breakthroughs comparing to the traditional substation [1] [2]. It greatly simplifies the deployment and maintenance of the substation, improves the reliability of the secondary system and provides the foundation of information sharing between various intelligent electronic devices, so that secondary equipment's interoperability and interoperability become easier. Meanwhile the digitalized system brings in new technical challenges, especially in the information analyzing field [3].
With the smart substation's information system getting fully digitalized and networked, the traditional connection terminals become invisible and intangible. Due to the invisibility of the connecting circuits, it is difficult for the substation operation and maintenance personnel to locate and troubleshoot the failures caused by the secondary and the network devices. Existing network packet recording products and solutions lack the ability to graphically represent and intelligently analyse the information interaction between IED devices. There is no sufficient solution for technical personnel, especially those with background experience of traditional substation's operation and maintenance, to locate, understand and resolve faults, therefore no guarantee for the safe and reliable operations of substations [4].

Aiming at the problem that the IED device interaction is not intuitionistic in the smart substation, a technology research on interpreting the process in an obvious way becomes extremely valuable.

\subsection{Technical Background}

IEC61850 is a standard for vendor-agnostic engineering of the configuration of Intelligent Electronic Devices for electrical substation automation systems to be able to communicate with each other. IEC 61850 is a part of the International Electro-technical Commission's (IEC) Technical Committee 57 (TC57) reference architecture for electric power systems. The abstract data models defined in IEC 61850 can be mapped to a number of protocols. Current mappings in the standard are to MMS (Manufacturing Message Specification), GOOSE (Generic Object Oriented Substation Event), SMV 
(Sampled Measured Values), and soon to wider messaging manners. These protocols can run over TCP/IP networks or substation LANs using high speed switched Ethernet to obtain the necessary response times below four milliseconds for protective relaying [1] [2].

In a standardized smart substation, IEDs interactive each other via three messaging mechanisms defined in IEC61850 :

GOOSE ( Generic Object Oriented Substation Events) is a controlled model mechanism in which any format of data (status, value) is grouped into a data set and transmitted within a time period of 4 milliseconds.

SMV ( Sampled Measured Value) is used for transmitting digitized instantaneous values of power system quantities, mainly primary currents and voltages. Thus, SMVs can replace the classical secondary quantities.

GOOSE packets and SMV packets are based on Layer 2 transmission, and are generally multicast. In addition to these two, there are MMS messages.

MMS (Manufacturing Message Specification) is an international standard (ISO 9506) dealing with messaging system for transferring real time process data and supervisory control information between networked devices and/or computer applications. The standard is developed and maintained by the ISO Technical Committee 184 (TC184). The IEC61850 standard absorbs and applies MMS to realize the exchange of the data and the monitoring information between the computer application program and the intelligent electronic device (IED) under the network environment by modelling the real equipment and its function.

With the gradual popularization and application of the smart substation, IEC61850 communication is widely used in substation automation system. The interaction of the devices in the system is changed, and the associations between the devices are established by networked message exchanges, rather than through the complex physical connections of the past. This aspect simplifies the system wiring, increases the overall reliability of the system, but on the other hand brings new challenges in substation monitoring, testing, maintenance, fault diagnosis, upgrade and so on [5][6].

\subsection{Visual Analysis of IED Interactive Processes}

The so-called IED interaction visual analysis is able to display in accordance with the timing of the interrelated IED messages, events and alarms, including SMV sampling, protection tripping, circuit breaker failure and other action events. Through the interaction of the entire event in the process tracking, no matter which part of the exception, the user can easily pinpoint it. The results of event tracking can be saved as templates, which can be intuitively compared with the results of the future experiments.

For example, when a fault happens, the switch is not the case of performing an expected operation. Current common method is to gradually check the merger unit output, then the protection of the output signal, then the smart terminal's received signals, etc. With the visual analyzing mechanism, all of the related events are automatically correlated together and displayed timesequentially. The misplayed step can be identified obviously.

\section{Preliminaries}

\subsection{Technical Basis}

(1) The model of the information to be displayed graphically is requested to be accurately described in the substation configuration profile (SCD) defined in XML format. Through the existing technology, we can extract all of the relevant information needed for correlating.

(2)The secondary circuit of the smart substation to be analysed is (optical or electrical) Ethernet networked. The services such as measurement, protection and remote control are based on the IEC61850-compliant information model, and utilized via IEC61850 standardized information exchange between different IEDs.

(3)It is a huge workload regarding the information collection in substation, effective information extraction and data storage. But based on the current technology it is achievable.

(4)The key of the problem addressed here is how to effectively correlate multiple valid information sources, such as the IED device names, event details, associated IED devices, the order of occurrence and so on, and what 
technology to be utilized to perform a good graphical representation.

(5)Java 2D provides a mechanism for implementing complex graphics that are well integrated with the Java platform's GUI architecture [7] [8]. It includes artistic lines, text, and images in a comprehensive model. It also provides a set of classes for precise colour space definition and transformation, as well as a large number of image symbols for display. Using Java 2D utilities, we can easily construct the data structure of the graph or network, and use classic algorithms (such as clustering, shortest path, maximum flow, etc.) to realize our own algorithms and graphically display the complex information. Facts have proved that Java 2D technology is sufficient enough to achieve the substation information presenting with high level of flexibility and user experiences.

\subsection{The graphing function of Java 2D technology}

A visualization mechanism to describe the interactive process of IEDs is introduced in this paper, which makes things easy to view the events and verify the secondary circuit in order to facilitate the operation and maintenance personnel to quickly pinpoint the abnormal.

In order to achieve the above purpose, we use Java2D technology as a concrete means of implementation.

(1) According to the information to be displayed, the IEDs involved are introduced into the graph as primitives, and can be rendered in different forms according to the IED type. The properties of the IEDs mainly include element shape, colour, and instantiation name of the IED device within the substation. If an IED or a DA that is not present in the SCD, it is marked as an unknown IED or unknown DA. Communication between the devices is represented as a directed line associated with the IED. The direction indicates the direction in which the packets are sent. The sequence number indicates the event occurrence time and the event ID and event type are rendered as well. An automatic layout algorithm is used to position the IEDs to gain the best overall presentation effect.

(2) Customizable playback of dynamic graphing. Substation events occur in the millisecond level, so real- time display is meaningless from analysing point of view. Therefore, through a certain technical means, while supporting real-time data processing; we need a mechanism to control the playback interval for manually progressive playback. By manually setting the graphing mode, we can select IED and other aspects involved in the charting. By adjusting time interval, we can control the speed of playing back to facilitate different reading purposes.

(3) For each result, we can view the details of the interaction via clicking on the directed lines. By clicking on a line segment, the IED device associated can be individually enlarged and down-to DA level detailed information can be retrieved and presented.

\section{The Implementation}

The visual analysing method described in this paper can represent interactive processes between protection relay devices, smart terminal, merging unit and other kind of secondary equipment, even including substation layer SCADA system and master station.

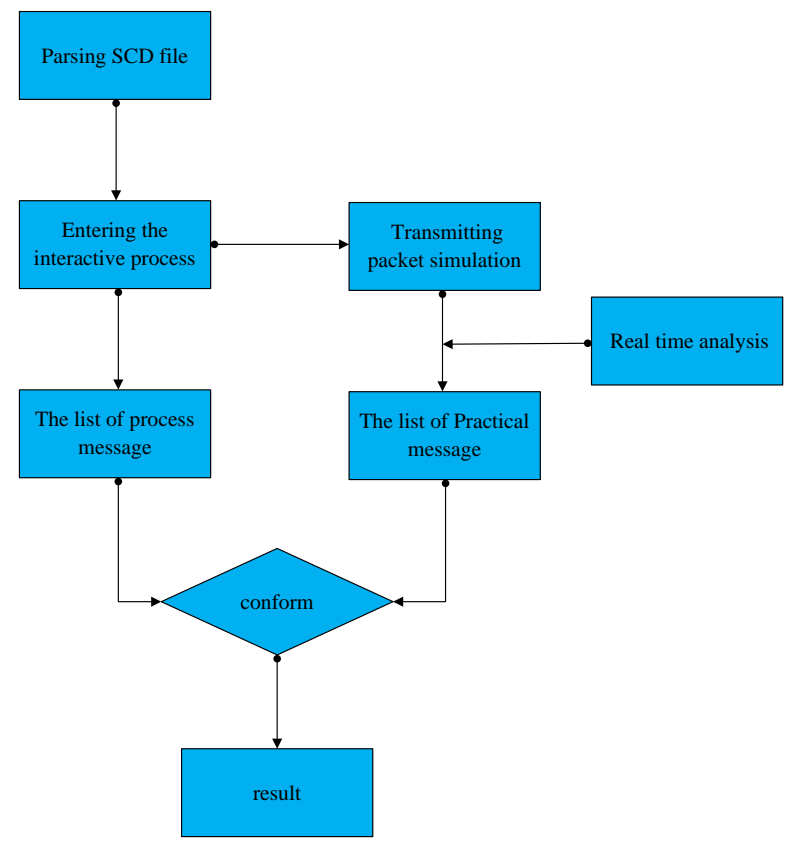

Figure 1 : System logic flow diagram

The main logic flow of implementation is shown below:

(1) Parsing SCD file :

According to the SCL schema defined by IEC61850, the JAXB JAVA library is used to parse and interpret the 
SCD file of the smart substation. The contents to be acquired include IED definitions, data set, Inputs, DO, DA, and other relevant description information.

The IED data set and Inputs are the data foundation of graphing, so well the detailed information regarding SMV, GOOSE, MMS and DA definitions.

(2) Classifying, Storing and Indexing SCD modelling information:

After parsing the SCD file, IED, SMV, GOOSE, MMS and other modelling information are classified and saved in the internal MySQL database. The indexes crossing database tables are established for fast data retrieving.

(3) Real-time substation messaging event acquirement:

After grabbing the substation communication traffic for a certain time period, the relevant messaging information is extracted via packet sniffing and filtering technology based on selected conditions. The captured messages include fault records, GOOSE actions and MMS report events, etc. The additional information includes event type, time of event occurrence, associated DA information, detailed description of event, IED names of all participating devices in the event, and other relevant.

The acquired contents are pre-processed, and saved into the "result queue" according to the order of occurrence of events.

(4) Graphical representation

According to the data in the "result queue", they are played back in chronological order. The IEDs involved are introduced asynchronously. Java2D technology is applied to complete the charting function. There are three major manners of graphical representation: message list mode, the dialogue mode and visual graphics mode. The list mode emphasizes the integrity of information. The dialogue mode emphasizes the interactive information and graphic mode emphasizes the graphical information. These three models show the whole process of the incident from different angles for the fault diagnosis and business logic verification.

(1) The List Mode

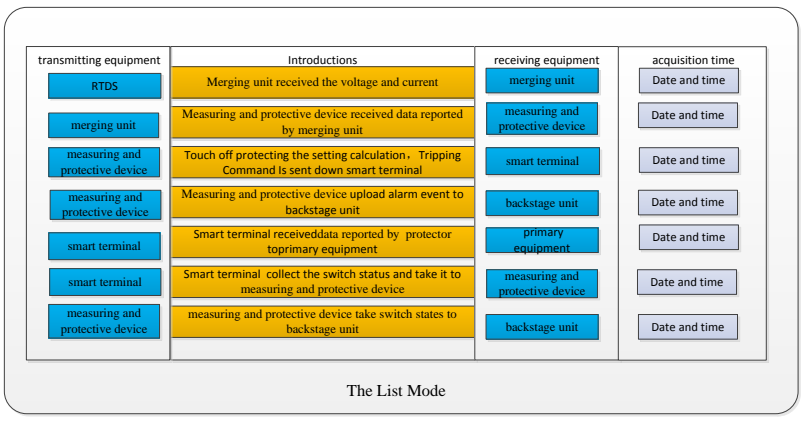

Figure 2: Sample of the list mode displaying

List mode is representing the course of events in a list with chronological order. Each piece of information corresponds to the message transmitting side device, event happening time, message receiving device, and time of the event on the receiving end.

(2) The Dialogue Mode

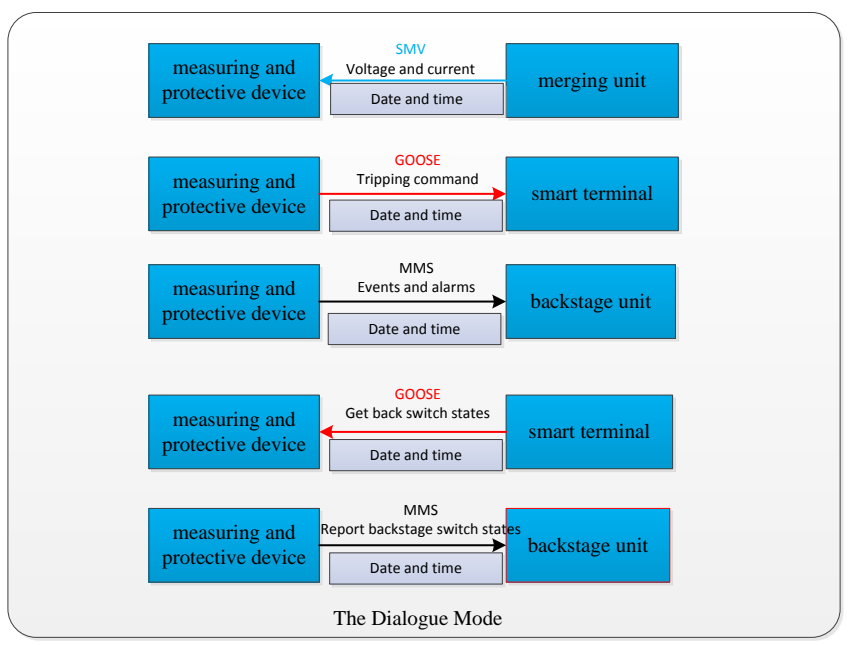

Figure 3 : Sample of the dialogue mode displaying

The dialogue mode makes the whole process of the event displaying more humanized. Through the interactive SYN-ACK way of expression, it represent the time of event happening, the details of the event, the transmitting and receiving devices with a user friendly manner.

(3) The Graphic Mode

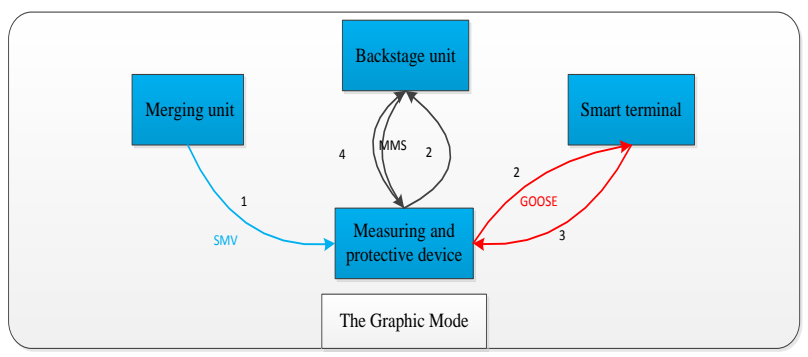

Figure 4: Sample of the graphic mode displaying 
The visual graph mode visually displays all IED devices with interaction related to this event, and can easily view the sequence of events and information flow direction.

\section{Main Problem addressed}

We proposed an effective means for the verification of the secondary circuit by recording and graphically displaying the interactive events between IEDs in smart substation.

The method can display the events for a period of time in an obvious way via multiple representation modes.

The method has been applied in more than ten substations in Fujian province of China, and effectively resolved some complex problems in the fault inspection and maintenance. For instance, it took hours to manually check the large amount of recorded network messages to identify the disoperation problem of protection before. Through our new mechanism, it only took a few minutes to locate the cause lies. The statistic results show an overall $40 \%$ improvement on efficiency of $\mathrm{O} \& \mathrm{M}$ in smart substation, which proves the strong practicability of the technique.

\section{Conclusion}

In this paper we have presented a graphical representation of the IED interactive process analysis in smart substation. Through parsing the SCD configuration file and extracting the relevant information needed, the data objects and their associations are classified, stored and indexed in the system internal database. While sniffing the mass communication data flow from the smart substation network, the related messaging data is effectively correlated. Combined with the data objects retrieved from the SCD configuration file and the messaging event information acquired, a Java 2D based unique solution is implemented to graphically represent dynamic interaction between IEDs in time sequenced manner, which greatly facilitates the rapid positioning of the failures by the substation technical personnel.

\section{References}

[1] IEC61850: Communication networks and systems for power utility automation, International Electro-technical Commission (IEC) Std., 2010.

[2] V. Gungor, D. Sahin, T. Kocak, S. Ergut, C. Buccella, C. Cecati, and G. Hancke, "Smart grid technologies: Communication technologies and standards", IEEE Transactions on Industrial Informatics, vol. 7, no. 4, pp. 529-539, 2011

[3] GB/T 30155-2013: Technical guide for smart substation [S]. Beijing, China standard publishing press, 2014.

[4] STATE GRID Corporation of China Q/GDW 689-2012 Commissioning specification for smart substation[S]. Beijing, China electric power press, 2012.

[5] Fan Chen, Ni Yimin, Dou Renhui, et al. Interpretation of relevant specifications of integrated supervision and control systems in smart substations $[\mathrm{J}]$. Automation of Electric Power Systems, 2012, 36(19):1-5

[6] Wang Lei, Sun Xiaofei. Major technical differences in the secondary system between smart substation and traditional substation [J]. Northeast Electric Power Technology, 2012, 33(2): 18-20.

[7] JIANG YI, ZHANG Hailong, WU Junjie, Gallery Electric Power System is the Integration of the Research. Power System and Clean Energy , 2016, 32 (4) ,85-88.

[8] F. Klawonn. Introduction to Computer Graphics: Using Java 2D and 3D (2nd edition). ISBN: 1447127323. 2012 